\title{
BMJ Open Cost-effectiveness of a care manager collaborative care programme for patients with depression in primary care: economic evaluation of a pragmatic randomised controlled study
}

\author{
Anna Holst, ${ }^{1}$ Annika Ginter, ${ }^{2}$ Cecilia Björkelund, ${ }^{1}$ Dominique Hange, ${ }^{1}$ \\ Eva-Lisa Petersson, ${ }^{1,3}$ Irene Svenningsson, ${ }^{1,3}$ Jeanette Westman, ${ }^{4}$ Malin André, ${ }^{5}$ \\ Carl Wikberg, ${ }^{1}$ Lars Wallin, ${ }^{6,7}$ Christina Möller, ${ }^{8}$ Mikael Svensson ${ }^{2}$
}

To cite: Holst A, Ginter A, Björkelund C, et al. Costeffectiveness of a care manager collaborative care programme for patients with depression in primary care: economic evaluation of a pragmatic randomised controlled study. BMJ Open 2018;8:e24741. doi:10.1136/ bmjopen-2018-024741

- Prepublication history for this paper is available online To view these files, please visit the journal online (http://dx.doi. org/10.1136/bmjopen-2018024741).

Received 11 June 2018 Revised 22 July 2018 Accepted 4 September 2018

Check for updates

(C) Author(s) (or their employer(s)) 2018. Re-use permitted under CC BY-NC. No commercial re-use. See rights and permissions. Published by BMJ.

For numbered affiliations see end of article.

Correspondence to

Dr Cecilia Björkelund;

cecilia.bjorkelund@allmed.gu.se

\section{ABSTRACT}

Objective To evaluate the cost-effectiveness of a care manager (CM) programme compared with care as usual (CAU) for treatment of depression at primary care centres (PCCs) from a healthcare as well as societal perspective.

Design Cost-effectiveness analysis.

Setting 23 PCCs in two Swedish regions.

Participants Patients with depression $(n=342)$.

Main outcome measures A cost-effectiveness analysis was applied on a cluster randomised trial at PCC level where patients with depression had 3 months of contact with a CM (11 intervention PCCs, $n=163$ ) or CAU (12 control PCCs, $n=179$ ), with follow-up 3 and 6 months. Effectiveness measures were based on the number of depression-free days (DFDs) calculated from the Montgomery-Åsberg Depression Rating Scale-Self and quality-adjusted life years (QALYs). Results were expressed as the incremental cost-effectiveness ratio: $\triangle$ Cost/ $\triangle Q A L Y$ and $\triangle \mathrm{Cost} / \triangle \mathrm{DFD}$. Sampling uncertainty was assessed based on non-parametric bootstrapping.

Results Health benefits were higher in intervention group compared with CAU group: QALYs (0.357 vs 0.333 , $p<0.001)$ and DFD reduction of depressive symptom score (79.43 vs $60.14, p<0.001$ ). The mean costs per patient for the 6-month period were $€ 368$ (healthcare perspective) and $€ 6217$ (societal perspective) for the intervention patients and $€ 246$ (healthcare perspective) and $€ 7371$ (societal perspective) for the control patients (n.s.). The cost per QALY gained was $€ 6773$ (healthcare perspective) and from a societal perspective the CM programme was dominant.

Discussion The CM programme was associated with a gain in QALYs as well as in DFD, while also being cost saving compared with CAU from a societal perspective. This result is of high relevance for decision-makers on a national level, but it must be observed that a CM programme for depression implies increased costs at the primary care level.

Trial registration number NCT02378272; Results.

\section{Strengths and limitations of this study}

- The study should be relevant both on a national healthcare level as well as at a clinical level, as mental health problems today constitute a growing part of healthcare costs at all levels and also affect health insurance costs.

- Both healthcare costs as well as societal costs were used for analyses, as societal costs widely exceed healthcare costs in the form of sick leave costs.

- We used robust and accepted methods for health economic analyses and modelling.

- By scrutinising electronic patient records extensive patient, care consumption, and sick leave data could be obtained.

- As in most cost-effectiveness studies of depression treatment, sick leave (absenteeism) was the measure used to estimate loss of productivity, but the cost of loss of productivity during depression is highly likely to be considerably underestimated, as presenteeism was not taken into account.

\section{BACKGROUND}

Depression is a major source of human suffering and a great and growing challenge for societies worldwide. ${ }^{1}$ Depression affects $10 \%-15 \%$ of the population. ${ }^{2}$ From an economic point of view, the disorder puts a high burden on affected individuals and also on society, including healthcare costs, sick leave and disability pension. ${ }^{3}$ The total annual cost for mood disorders in Europe 2010 was estimated to approximatively $€ 113$.4 billion, which corresponds to almost $1 \%$ of the gross domestic product in the European Union. ${ }^{4}$

The majority of people with depressive symptoms seek care and are treated in primary care. ${ }^{125}$ However, recommendations and guidelines for depression treatment are 
mainly based on research at the psychiatric, secondary care, level. ${ }^{2}$ In order to provide access to the most effective care for depression, new evidence-based treatment methods and organisational forms of care need to be evaluated at the primary care level. International studies conclude that isolated actions such as increased screening for depression, special training of doctors and nurses or increased psychological expertise in primary care in itself do not result in higher quality of care or better effect than care as usual (CAU). ${ }^{67}$

Currently, best evidence internationally for high quality care and effectiveness of treating depression supports collaborative care with a care manager. ${ }^{8-10}$ A care manager provides continuous supporting contact with the patients including behavioural activation, follow-up and feedback regarding the patients' progress to the doctor and the primary healthcare team. An important function of the care manager is also to facilitate the engagement of the patients in their care through self-management support. ${ }^{810}$ The Swedish Council on Health Technology Assessment has stressed that studies on collaborative care with a care manager organisation in primary care need to be conducted in Sweden to evaluate the effect of this intervention in a Swedish context, where primary care mostly is organised in group practices also with specialised nurses, physiotherapists and psychotherapists, and triage systems. Consequently, the randomised controlled trial (RCT) PRIM-CARE was performed in Sweden 2014-2016, which compared collaborative care with a care manager to CAU as treatment for depression in the primary care setting. ${ }^{11}$ The results showed that a care manager organisation at the primary care centre (PCC) has positive effects on patients with depression regarding depression course, remission frequency, return to work and quality of life compared with CAU.

A large amount of evidence shows that besides having positive effects on symptom reduction and quality of care, this type of intervention also is cost-effective. ${ }^{12}$ However, in a systematic review of enhanced primary care for treating depression, Gilbody et al concluded that improved outcomes are expected for collaborative care, but at an increased cost that will require investments. ${ }^{13}$ At present there are no Swedish studies on cost-effectiveness of a care manager programme for treatment of depression in Swedish primary care context.

\section{AIM}

The aim of this study was to evaluate the cost-effectiveness of a care manager programme compared with CAU for treatment of mild to moderate depression in the Swedish primary care setting.

\section{METHOD}

\section{Study design}

A commonly used form of health economic evaluation is cost-effectiveness analysis (CEA). CEA evaluates the effects/benefits of a healthcare intervention and one or more alternative options in relation to their costs. The results serve as guidance for decision-makers in order to allocate scarce healthcare resources most efficiently. ${ }^{14}$ In this study, two effectiveness measures were used: depression-free days (DFDs), which was calculated based on scores from symptoms expressed in changes on the Montgomery-Åsberg Depression Rating Scale-Self assessment (MADRS-S) ${ }^{15}$ and quality-adjusted life years (QALYs) ${ }^{16}$ The results of a CEA are expressed in the incremental cost-effectiveness ratio (ICER), which is the difference in costs divided by the difference in effectiveness of implementing the care manager programme compared with CAU: ICER $=\left(\right.$ Cost $_{\text {care manager }}-$ Cost $\left._{\mathrm{CAU}}\right) /\left(\right.$ Effectiveness $_{\text {care }}$ -Effectiveness ${ }_{\mathrm{CAU}}$ ). The following two ICERs were calculated in this study: $\Delta$ Cost $/ \triangle \mathrm{QALY}$ and $\Delta \mathrm{Cost} / \Delta \mathrm{DFD}$. The cost-effectiveness of the intervention was assessed at 6 months follow-up.

\section{PRIM-CARE}

The CEA was based on primary data collected from the pragmatic cluster RCT PRIM-CARE using PCCs as the level of randomisation. ${ }^{11}$ It can be seen as a pragmatic (randomised controlled) effectiveness trial, which is generally regarded as the best vehicle for CEA. ${ }^{17}$ The study was performed at 23 Swedish PCCs in the Regions Västra Götaland and Dalarna between December 2014 and January 2016 and included 376 patients with newly diagnosed mild to moderate depression $(<1$ month, according to MADRS-S <35). The PCCs were randomised into two groups: intervention $(n=11)$ and control $(n=12)$, where intervention patients $(n=192)$ received care manager contact during 3 months and control patients $(n=184)$ received CAU. The main outcomes of PRIM-CARE were patients' depressive symptoms measured by MADRS-S and Beck Depression Inventory II (BDI-II) ${ }^{18}$ patients' quality of life (assessed by EuroQoL five-dimension three-level (EQ-5D-3L) scale ${ }^{19}$ (weighted UK time trade-off values)), sick leave days and return to work, service satisfaction and antidepressant medication. Patients were assessed at baseline, 3 and 6 months.

\section{The intervention}

Intervention PCCs each established a nurse as care manager, who used $20 \%-25 \%$ of her/his working time to coordinate and manage care and support of patients with depression. Before the trial started, participating staff members were educated according to their tasks within the care manager programme (2 days for general practitioners (GPs), 5 days for nurses/care managers). Programme services for participating patients included an individual care plan (1-hour session per patient with care manager), regular telephone contacts between care manager and patient in order to assess self-rated depressive symptoms (at least 6-8 times during the 12-week intervention period), as well as the opportunity to contact the care manager at any point of unscheduled time if needed. Furthermore, care managers were in constant dialogue 
with GPs, therapists and other healthcare personnel in order to follow up patients' development. Thus, they did not perform any psychotherapeutic measures beyond behavioural activation and functioned as a supportive link between specialists and patients while improving accessibility and continuity of care, as well as treatment adherence.

In addition, care managers had regular follow-up meetings (every second month) during the study, where difficulties as well as successes were discussed together with the research team and the region's implementation team.

\section{Care as usual}

CAU could consist of visits to a GP, nurse, antidepressants, face-to-face psychotherapy (or being on the waiting list for such psychotherapy), sick listing or combinations of these.

\section{Outcome measures}

Main outcome measures were DFDs calculated based on depressive symptoms expressed as change in MADRS-S and QALYs based on EQ-5D-3L scores (weighted time trade-off values) assessed using the Dolan tariff. The number of DFDs was assessed by estimating the number of days each patient scored equal or below 12 on the MADRS-S. Considering that we have data from each patient at baseline, 3 and 6 months, linear interpolation was carried out between the measurement points to predict a MADRS-S score for each day. Additionally, sensitivity analysis was carried out calculating DFDs based on responses to the BDI-II instrument (depression free assumed at a score equal to or below 9). The same linear interpolation used to calculate DFDs between the measurement points was also carried out for the EQ-5D-3L scores to be able to calculate the QALYs for each patient.

\section{Cost measurements}

Costs were estimated both from a healthcare perspective taking into account the healthcare costs and from a societal perspective including the healthcare costs plus the costs due to loss of productivity. The currency of reference was Swedish Krona, corresponding to $\sim € 0.1$. Costs were measured in Swedish kronor (SEK) and based on the 2016 price level, but throughout the manuscript, we also present the main results in Euro.

All costs were obtained from primary data collected via electronic patient records (EPRs) and patient research interviews in the RCT and then linked to market prices. Healthcare costs included education costs for PCC personnel (only for the intervention group), contacts with healthcare professionals (physical and via telephone) and medication (meaning mostly antidepressants). Since Sweden has a publicly administered healthcare system, where professionals are employed by the counties, costs per healthcare contact and for staff education were calculated by means of time spent and gross wages (including social fees) of the respective professional groups. There was no inpatient care cost for this patient group. Consumption of pharmaceuticals was recorded per patient during the follow-up period and was then linked to Swedish market prices, derived from the Swedish Pharmaceutical Industry Association's Service (LIF).${ }^{20}$ Costs for loss of productivity were calculated by means of the human capital approach, ${ }^{14}$ using registered sick leave days (percentage-wise) during the follow-up period and the average gross wage (including social fees) for women in Sweden (since almost two-thirds of the study population were female). Given the short follow-up period, discounting was not applied.

\section{Analysis of cost-effectiveness}

The ICER was calculated as the ratio of differences in mean costs per patient and mean QALYs $\left(\frac{\triangle \text { Costs }}{\triangle Q A L Y s}\right)$ or mean DFDs per patient $\left(\frac{\triangle \text { Costs }}{\triangle D F D}\right)$, respectively, between the intervention group and the CAU group at 6 months follow-up. Considering that the design was a cluster randomised study, the differences in effectiveness and costs were analysed using a multilevel model where patients were nested within the PCCs. Patients were included (342 of 376) if data were available for baseline and at least one follow-up assessment. Missing values at the 3 or 6 months follow-up were imputed using linear regression analysis using non-missing EQ-5D-3L data together with individual characteristics (age, sex, education level, ethnicity, marital status) as predictors. Nine per cent of the randomised patients (34 out of 376) dropped out just after randomisation. Analysing the patients lost after randomisation indicated that the only significant predictor was age (sex, educational level, marital status, number of children, smoking, use of snuff, whether taking any antidepressant medication, were not at all related to dropping out of the study); where increasing in age by 1 year increased probability of missing by $0.4 \%$. Data analysis was carried out in Microsoft Excel and Stata V.15. Statistical significance was accepted at $\mathrm{p}<0.05$.

Sampling uncertainty was assessed using non-parametric bootstrapping focusing on the cost per QALY, which is the primary outcome measure in health economic evaluations and therefore facilitates the widest comparisons. ICERs for both effectiveness measures were estimated based on 5000 bootstrap resamples and summarised in a cost-effectiveness plane (CE-plane) and in a cost-effectiveness acceptability curve (CEAC). For the CE-plane, we show confidence ellipses showing the area containing $95 \%, 75 \%$ and $50 \%$ of the bootstrapped ICERs, together with the point estimate from the main analysis. In cases where the ICER results in a negative value, it is difficult to tell whether it is located in the Northwest quadrant of the CE-plane (less effective and more costly, referred to as a 'dominated treatment') or in the preferable Southeast quadrant of the CE-plane (more effective and less costly, referred to as a 'dominant treatment'). We addressed this potential confusion by estimating the net monetary benefit (NMB) instead of the ICER, which was subsequently used to construct the CEAC. The NMB is calculated using a different assumption of the monetary value of a QALY, that is, how much the decision-maker is willing to 
Table 1 Baseline characteristics for primary care patients in the PRIM-CARE RCT; intervention group (care manager) and control group (CAU)

\begin{tabular}{|c|c|c|c|c|}
\hline & Intervention $\mathrm{n}=192$ & Control $n=184$ & Total $\mathrm{n}=\mathbf{3 7 6}$ & $P$ values \\
\hline \multicolumn{5}{|l|}{ Age } \\
\hline Mean (SD) & $40.8(15.0)$ & $41.6(15.4)$ & $41.2(15.2)$ & 0.61 \\
\hline \multicolumn{5}{|l|}{ Gender, $\mathrm{n}(\%)$} \\
\hline Women & $131(68.2)$ & $137(74.5)$ & $268(71.3)$ & 0.18 \\
\hline Men & $61(31.8)$ & $47(25.5)$ & $108(28.7)$ & \\
\hline \multicolumn{5}{|l|}{ Occupation, $\mathrm{n}(\%)$} \\
\hline Working & $137(72.9)$ & $122(66.3)$ & $259(69.6)$ & \\
\hline Studying & $18(9.6)$ & $19(10.3)$ & $37(9.9)$ & \\
\hline In search of work/other & $23(17.6)$ & $43(23.4)$ & $76(20.5)$ & 0.52 \\
\hline \multicolumn{5}{|l|}{ Working, $\mathrm{n}(\%)$} \\
\hline Full-time & $157(87.7)$ & $149(87.6)$ & $306(87.7)$ & 0.98 \\
\hline Other $(25 \%-75 \%)$ & $22(12.3)$ & $21(12.4)$ & $43(12.3)$ & \\
\hline \multicolumn{5}{|l|}{ Marital status, $\mathrm{n}(\%)$} \\
\hline Cohabiting & $122(67)$ & $122(68)$ & $244(67)$ & 0.82 \\
\hline Single & $61(33)$ & $58(32)$ & $119(33)$ & \\
\hline Born outside of Nordic country, n(\%) & $18(9.4)$ & $21(11.5)$ & $39(10.4)$ & 0.63 \\
\hline \multicolumn{5}{|l|}{ Educational level, n(\%) } \\
\hline Primary education & $17(8.9)$ & $27(14.8)$ & $44(11.8)$ & \\
\hline Secondary education & $103(53.9)$ & $90(49.2)$ & $193(51.9)$ & \\
\hline University & $71(37.2)$ & $66(36.1)$ & $137(36.6)$ & 0.21 \\
\hline Sick leave at baseline, $n(\%)$ & $93(50.5)$ & $94(55.0)$ & $187(52.7)$ & 0.40 \\
\hline \multicolumn{5}{|l|}{ Health status } \\
\hline MADRS-S m(SD) & $20.8(7.2)$ & $21.9(7.1)$ & $21.4(7.1)$ & 0.12 \\
\hline $\mathrm{BDI}-\mathrm{II} \mathrm{m}(\mathrm{SD})$ & $23.9(8.7)$ & $25.1(8.5)$ & $24.5(8.7)$ & 0.16 \\
\hline EQ-5D m(SD) & $0.58(0.24)$ & $0.56(0.25)$ & $0.57(0.24)$ & 0.41 \\
\hline
\end{tabular}

Figures indicate numbers and percentage (\%) of patients.

BDI-II, Beck Depression Inventory II; CAU, care as usual; EQ-5D, EuroQoL five-dimension; MADRS-S, Montgomery-Åsberg Depression Rating Scale-Self; RCT, randomised controlled trial.

pay for a gain of 1 QALY. The formula to calculate the NMB is: $\triangle Q A L Y \times$ Value per $Q A L Y-\triangle$ Cost. An intervention is considered cost-effective as long as the NMB is positive, since this indicates that the costs to achieve the health benefits are below the respective willingness-to-pay threshold. ${ }^{14}$

\section{Patient and public involvement}

No patients were involved in the development of the research question or outcome measures, nor in the recruitment to or conduct of the study. The results will be disseminated to study participants through news media.

\section{RESULTS}

Baseline characteristics of the study population are presented in table 1 .

\section{Cost outcome}

A detailed overview of identification, valuation and distribution of costs can be seen in table 2. From a healthcare perspective, total cost per patient for the intervention group during the 6 months follow-up period were 3674 SEK (€368). Adding the costs for loss of productivity resulted in a cost per patient of 62174 SEK (€6217). For patients assigned to the control group, the corresponding values were 2464 SEK (€246) per patient and 73705 SEK $(€ 7371)$ per patient (table 2$)$. In both groups the greatest share of healthcare costs was related to contacts with healthcare professionals $(60 \%$ in the intervention group and $77 \%$ in the control group). Medication (mostly antidepressants) accounted for $15 \%$ of total healthcare costs in the intervention group and $23 \%$ in the control group. Education costs of personnel at intervention PCCs represented $25 \%$ of total healthcare costs. When considered from a societal perspective, costs for loss of productivity accounted for $94 \%$ of total costs in the intervention group and $96 \%$ in the control group. Distribution of healthcare costs among the two groups was rather similar. The most remarkable differences were observed in visits to and phone contacts with the nurse (due to the nature of the 
Table 2 Cost items, volumes used, prices per unit and average cost per patient

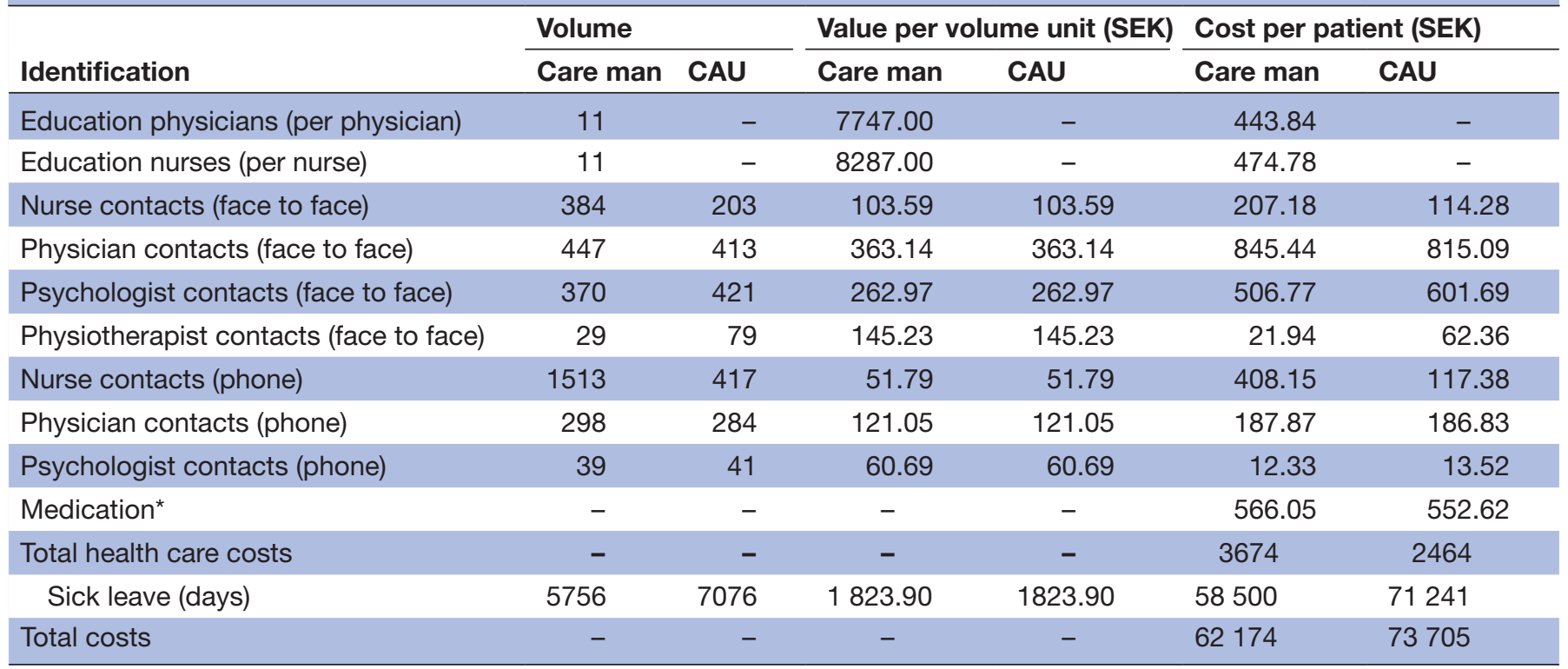

Data for all 376 randomised patients.

*Amounts of pharmaceuticals consumed were calculated individually per patient, according to prescription records during the study. Prices were obtained from a national pharmaceutical register (LIF) and then individually assigned to each preparation.

CAU, care as usual; SEK, Swedish kronor (approx. 1 SEK= €0.1).

intervention) and education costs, which were likewise only related to the care manager programme. Difference in mean costs between the two groups was not statistically significant $(\mathrm{p}=0.19)$.

\section{Health outcome}

As seen in the mid-part of table 3, health benefits were higher in the intervention group compared with the CAU group regarding both QALYs $(0.357$ vs 0.333$)$ and DFDs (79.43vs 60.14). Both differences showed statistical significance with $\mathrm{p}<0.001$. Sensitivity analyses based on calculating DFDs from the BDI-II instrument produced qualitatively similar results (2\% difference compared with the results shown in table 3 ).

\section{Cost-effectiveness}

From a societal perspective, the care manager programme dominated CAU, that is, it produced larger health benefits to a lower cost. From a healthcare perspective the cost per QALY was $€ 6773$ and the cost per DFD was $€ 7$ (table 3).

Table 3 Mean healthcare and societal costs per patient, as well as difference between care manager and care as usual (CAU) group in the PRIM-CARE RCT with $95 \% \mathrm{Cl}$

\begin{tabular}{|c|c|c|c|}
\hline Costs in Swedish kronor / € & Care manager & CAU & Adjusted difference $(95 \% \mathrm{Cl})^{*}$ \\
\hline Health care costs & 3674 / €368 & 2464 / €246 & $1210 / € 121$ (569 to 1852$)$ \\
\hline Societal costs & $58500 / € 5850$ & $71241 / € 7124$ & $-11531 / €-1153$ (-37 690 to 14627$)$ \\
\hline Total costs & 62174 / €6 217 & 73705 / €7371 & -11945 / €-2001 (-38 010 to 14120$)$ \\
\hline \multicolumn{4}{|l|}{ Patient outcome measures } \\
\hline QALYs & 0.357 & 0.333 & $0.018+(0.016$ to 0.019$)$ \\
\hline Depression Free Days (DFD) & 79.43 & 60.14 & $17.16 \dagger(3.84$ to 30.47$)$ \\
\hline \multicolumn{4}{|c|}{ Incremental cost-effectiveness ratios in Swedish kronor (SEK) / € } \\
\hline Cost per QALY: societal perspective & & \multicolumn{2}{|c|}{ Care manager is dominant } \\
\hline Cost per QALY: health care perspective & & \multicolumn{2}{|c|}{67731 SEK / €6773 } \\
\hline Cost per DFD: societal perspective & & \multicolumn{2}{|c|}{ Care manager is dominant } \\
\hline Cost per DFD: health care perspective & & \multicolumn{2}{|l|}{71 SEK / €7 } \\
\hline
\end{tabular}

${ }^{*} 95 \% \mathrm{Cl}$ is adjusted for the fact that patients are clustered within primary care centres and difference estimates are adjusted for baseline data on health status.

†P value for difference in mean $<0.001$.

$\mathrm{RCT}$, randomised controlled trial; QALY, quality-adjusted life year. 

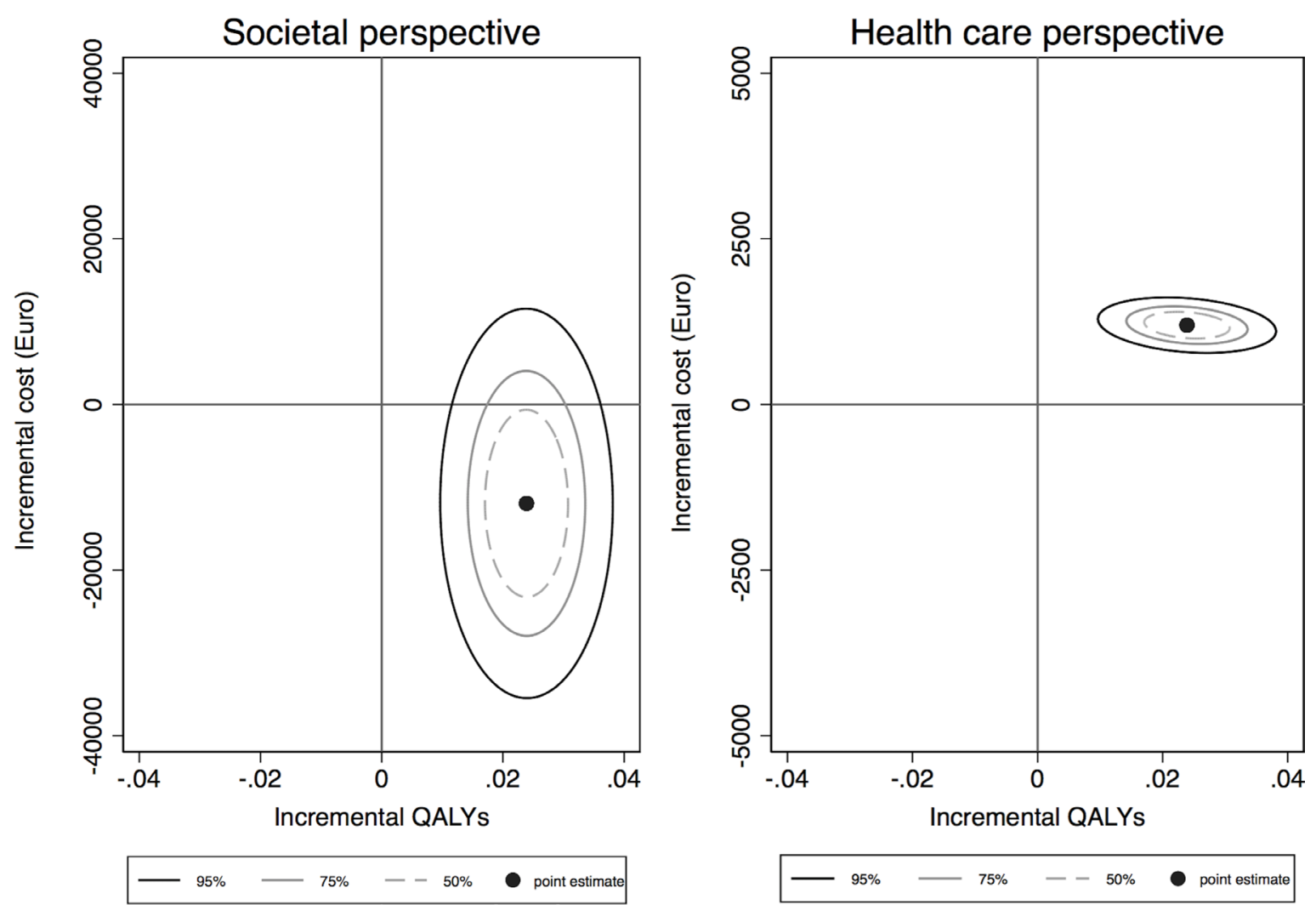

Figure 1 Cost-effectiveness planes with confidence ellipses. The horizontal axis represents the difference in QALYs between the care manager programme and CAU. The vertical axis represents the difference in costs between the two alternatives (left graph: societal perspective, right graph: healthcare perspective). CAU, care as usual; QALYs, quality-adjusted life years.

The bootstrapped ICERs drawn from the study sample are presented in the form of cost-effectiveness ellipses on the CE-plane in figure 1. From a societal perspective, most ICERs are in the Southeast quadrant of the CE-plane, which indicates that the care manager programme is likely to be more effective and less costly compared with CAU. From a healthcare perspective, most ICERs are in the Northeast quadrant, although at a relatively low increasing cost per QALY, indicating that the care manager programme increases costs at the same time as it improves health.

The CEAC in figure 2 shows the probability of the care manager programme being cost-effective for several willingness-to-pay thresholds per QALY (in euros). At a willingness-to-pay threshold of $€ 10000$ per QALY, there was a $93 \%$ probability of the care manager programme being cost-effective from a societal perspective and $97 \%$ likelihood that it was cost-effective from a healthcare perspective (higher due to less variability).

\section{DISCUSSION}

This health economic evaluation showed that health benefits were statistically significantly greater in a PCC care manager organisation for patients with depressive disorder compared with CAU regarding both QALYS and DFDs. Healthcare costs differed to the advantage of CAU, but the difference in total costs between the two groups was not significantly different. The cost-effectiveness analysis showed that from a societal perspective the care manager programme dominated CAU by leading to higher health benefits and lower costs. From a healthcare perspective the cost per QALY gained was $€ 6773$ and the cost per DFD was $€ 7$. Already at a willingness to pay per QALY of $€ 10000$, it was $93 \%$ (societal)

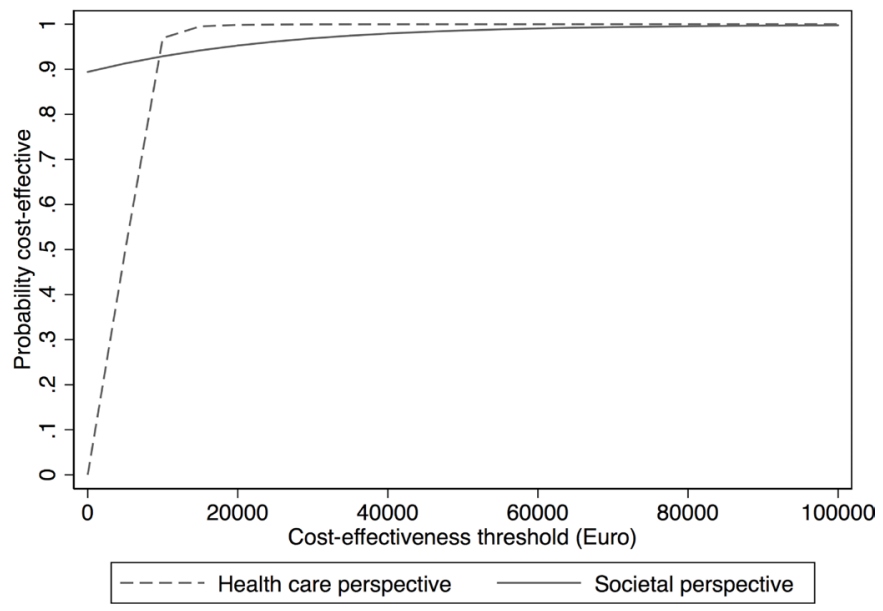

Figure 2 Cost-effectiveness acceptability curves for various willingness-to-pay thresholds for one quality-adjusted life year gained based on a healthcare and societal perspective. 
to $97 \%$ (healthcare) likelihood that the care manager programme was cost-effective.

\section{Strengths and limitations of this study}

This health economic evaluation of an organisational intervention has several strengths. The study is relevant both on a national healthcare level as well as a societal level, as mental health problems today constitute a growing part of healthcare costs, especially at the primary care level, and also affect health and social insurance costs. Among the strengths are the extensive patient, care consumption and sick leave data obtained by examining EPRs in addition to retrieving data from the patients and the PCCs' personnel.

Participating patients were acceptably diversified in age and gender. Both patients and PCCs showed very good participation rates, partly due to support from the study group, which had thorough experience from primary care and accomplishment of primary care clinical trials. We used both healthcare costs as well as societal costs for our analyses, as societal costs widely exceed healthcare costs in the form of sick leave costs. We used robust and accepted methods for health economic analyses and modelling. The results may be regarded as generalisable and representative for Swedish primary care.

A limitation was the follow-up time, which was only 6 months. Health economic consequences with regard to healthcare consumption, health status and sick leave should preferably be assessed within a longer time perspective. This will be done further on when data from a long-term follow-up become available. However, it should be noted that it is likely that the care manager programme would be even more cost-effective with a longer follow-up time, considering that the improved health was maintained also at 6 months.

In this study, as well as in most cost-effectiveness studies of depression treatment, sick leave (absenteeism) ${ }^{21}$ was the measure used to estimate loss of productivity, and which also represented the largest societal cost for depression. However, patients with depression are usually present at work, but their performance can be substantially reduced because of their state (presenteeism). ${ }^{21}$ As much as $81 \%$ of the productivity loss cost could be explained by reduced performance while at work during depression. ${ }^{21}$ The cost of loss of productivity during depression is highly likely to be considerably greater than currently measured, as presenteeism was not taken into account.

\section{Findings in relation to other studies}

Due to differences in healthcare systems including aspects such as professional roles, resources, access to healthcare or organisational levels of care, comparison between cost-effectiveness studies is limited. Moreover, included cost categories and health effects may differ. Nevertheless, the results in the present study are in line with the overall results in the literature. The systematic review of Gilbody et al showed that the majority of the included economic evaluations from the USA found positive health effects as well as increased healthcare costs associated with the intervention. ${ }^{13}$ ICERs varied between US $\$ 15463$ and US $\$ 36467$ (€13 138 and $€ 30984$ ) and were located in the Northeast quadrant of the CE-plane (that is, intervention is effective but more costly compared with CAU). This might be due to the fact that none of the studies included societal costs such as loss of productivity. A more recent systematic review showed incremental costs per QALY from dominant (located in the Southeast quadrant of the CE-plane, that is, intervention is more effective and less costly) to US $\$ 874562$ (€743 059 ) but only 5 out of 19 studies had used a societal perspective. ${ }^{22}$ Since our study considered costs from a societal perspective, direct comparisons are not possible here. Nonetheless, our results indicated larger health benefits and lower costs, yielding more favourable results in terms of cost-effectiveness. The Clinical and Cost Effectiveness of Collaborative Care for Depression in UK Primary Care Trial (CADET) study had an estimated mean cost per QALY of $£ 14248$ (€16 236) but included no costs for loss of productivity. ${ }^{23}$

Gilbody et al noted that a societal perspective is more meaningful to policy-makers and that there is evidence for collaborative care programmes having positive effects on sick leave. ${ }^{13}$ The latter study's results correspond to our findings. Furthermore, $70 \%$ of the current study population were in the work force, indicating that a societal perspective was of high relevance for this study.

More recent evaluations have accounted for societal costs and are therefore more suitable for comparison. Aragonès $e t$ al found in Spain that the collaborative care programme INDI was cost-effective $($ ICER $=$ US $\$ 4056$ per QALY). Nonetheless, due to only small differences in sick leave days between the study groups, total costs in the intervention group were still higher than the ones in the control group. ${ }^{24}$ This located the ICER in the Northeast quadrant of the CE-plane (INDI more effective and more costly than CAU). On the other hand, the results of a German study were similar to ours, meaning that total costs for the control group exceeded total costs for the intervention group, when loss of productivity was included. The ICER for total societal costs was not clearly stated, but the tables suggested an ICER of $€ 66092$ per QALY. Our more favourable result is mainly due to lower costs in nearly all cost categories. Effects regarding QALYs were almost identical to our study. ${ }^{25}$ Both of the studies identified the societal costs as the biggest share of total costs, which was also the case in our study.

\section{Significance of the study}

The evaluation of interventions that can facilitate the implementation of evidence-based care for patients with depression in primary care is of great importance, as there is an identified knowledge gap in this area. ${ }^{6}$ To assess the cost-effectiveness of an intervention is crucial, as the societal as well as the healthcare resources are limited, and decision-makers need thorough documentation to be able to prioritise between different options. In primary care, the cornerstones of high quality care are 
accessibility and continuity, aspects that are promoted by care adjusted to the individual's needs and also support of the individual's capacity to manage the illness during the course of the rehabilitation. A care organisation at the PCC, where the care manager is the hub and facilitates both the patient's contacts with healthcare and the collaborative care model within the PCC, can be effective in several ways, both for the patient, for the primary care unit and for society at large. This health economic evaluation confirms beneficial effects on several levels that can be useful for policy-makers as well as for clinicians.

\section{Implications for healthcare}

The high incidence of depression makes it important to evaluate and implement new effective forms of care. This study shows that a care management organisation at PCCs is beneficial for patients with depression as well as for the national economic system. However, the major benefits are obtained on a societal level, while the costs ( $14 \%$ increase) for increasing quality of care and effectiveness are generated on the healthcare level. As a next step, the Swedish authorities should evaluate whether a nationwide implementation of the care manager programme is feasible. In case of feasibility, the financing of the implementation should include transformation of the societal health insurance (monetary) gain to (primary) healthcare level. Unlike some other countries, Sweden does not have an 'official' threshold to determine whether an intervention should be implemented. However, there is an informal rule which considers any intervention below 500000 SEK per QALY ( $€ 50000$ ) as cost-effective, substantially higher than the estimate reported in this study. ${ }^{26}$

\section{CONCLUSIONS}

The results of this study indicate that a collaborative care programme involving a care manager organisation for patients with depression is highly cost-effective in a primary care setting over a follow-up period of 6 months. From a societal perspective, the programme is dominant for both effectiveness measures-DFDs and QALYswhich means that it generates higher health benefits for the patient at lower costs compared with usual primary care of today. This result is of high relevance for decision-makers on a national level. It is further noteworthy that the care manager programme has low implementation costs (education of PCC personnel), which may result in even higher cost-effectiveness in the future.

\footnotetext{
Author affiliations

${ }^{1}$ Department of Public Health and Community Medicine/Primary Health Care, Institute of Medicine, Sahlgrenska Academy, University of Gothenburg, Gothenburg, Sweden

${ }^{2}$ Department of Public Health and Community Medicine/Health Metrics, Institute of Medicine, Sahlgrenska Academy, University of Gothenburg, Gothenburg, Sweden ${ }^{3}$ Närhälsan Research and Development Primary Health Care, Region Västra Götaland, Gothenburg, Sweden

${ }^{4}$ Division of Family Medicine, Department of Neurobiology, Care Sciences and Society, Karolinska Institute, Stockholm, Sweden
}

${ }^{5}$ Department of Public Health and Caring Sciences-Family Medicine and Preventive Medicine, Uppsala University, Uppsala, Sweden

${ }^{6}$ Division of Nursing, Department of Neurobiology, Care Sciences and Society, Karolinska Institute, Stockholm, Sweden

${ }^{7}$ Department of Health and Care Sciences, Sahlgrenska Academy, University of Gothenburg, Gothenburg, Sweden

${ }^{8}$ Primary Health Care Head Office, Närhälsan, Region Västra Götaland, Hisings Backa, Sweden

Contributors AH, MS, CB, AG, DH, IS, E-LP, JW, AM, CW, LW and CM participated in the design of the study. AH, MS, CB and AG handled and analysed data. AH, MS, $\mathrm{CB}, \mathrm{AG}, \mathrm{DH}, \mathrm{IS}, \mathrm{E}-\mathrm{LP}, \mathrm{JW}, \mathrm{AM}, \mathrm{CW}$, LW and CM drafted and revised the paper. CB is chief investigator and initiated the project, chaired trial management group and is guarantor. All authors had full access to all of the study data and take responsibility for the integrity and accuracy of the data. All authors have read and approved the final version of the paper.

Funding This work was supported by grants from Region Västra Götaland, Sweden. Competing interests None declared.

Patient consent Not required.

Ethics approval Ethical approval was given by the Regional Ethical Review Board in Gothenburg, Sweden (Dnr 903-13; 2 January 2014).

Provenance and peer review Not commissioned; externally peer reviewed.

Data sharing statement The datasets generated during and/or analysed during the current study are not publicly available due to Swedish law, but are available from the corresponding author on reasonable request.

Open access This is an open access article distributed in accordance with the Creative Commons Attribution Non Commercial (CC BY-NC 4.0) license, which permits others to distribute, remix, adapt, build upon this work non-commercially, and license their derivative works on different terms, provided the original work is properly cited, appropriate credit is given, any changes made indicated, and the use is non-commercial. See: http://creativecommons.org/licenses/by-nc/4.0/.

\section{REFERENCES}

1. Pelone F, Kringos DS, Spreeuwenberg P, et al. How to achieve optimal organization of primary care service delivery at system level: lessons from Europe. Int J Qual Health Care 2013;25:381-93.

2. Swedish Council on Technology Assessment in Health Care. Behandling av depressionssjukdomar. En systematisk litteraturöversikt.Treatment of depression. A systematic review. Stockholm: Swedish Council on Technology Assessment in Health Care, 2004

3. Ekman M, Granström O, Omérov S, et al. The societal cost of depression: evidence from 10,000 Swedish patients in psychiatric care. J Affect Disord 2013;150:790-7.

4. Olesen J, Gustavsson A, Svensson M, et al. The economic cost of brain disorders in Europe. Eur J Neurol 2012;19:155-62.

5. Bower P, Rowland N, Hardy R. The clinical effectiveness of counselling in primary care: a systematic review and meta-analysis. Psychol Med 2003;33:203-15.

6. Swedish Council on Health Technology Assessment. Implementeringsstöd för psykiatrisk evidens i primärvården. En systematisk litteraturöversikt. Implementation support for the psychiatric evidence in primary care. A systematic review. Stockholm: Swedish Council on Health Technology Assessment, 2012.

7. Gilbody S, Whitty P, Grimshaw J, et al. Educational and organizational interventions to improve the management of depression in primary care: a systematic review. JAMA 2003;289:3145-51.

8. Archer J, Bower P, Gilbody S, et al. Collaborative care for depression and anxiety problems. Cochrane Database Syst Rev 2012;10:CD006525.

9. Rost K, Nutting P, Smith J, et al. Improving depression outcomes in community primary care practice: a randomized trial of the quEST intervention. quality enhancement by strategic teaming. J Gen Intern Med 2001;16:143-9.

10. Goodrich DE, Kilbourne AM, Nord KM, et al. Mental health collaborative care and its role in primary care settings. Curr Psychiatry Rep 2013;15:383.

11. Björkelund C, Svenningsson I, Hange D, et al. Clinical effectiveness of care managers in collaborative care for patients with depression 
in Swedish primary health care: a pragmatic cluster randomized controlled trial. BMC Fam Pract 2018;19:28.

12. van Steenbergen-Weijenburg KM, van der Feltz-Cornelis CM, Horn $\mathrm{EK}$, et al. Cost-effectiveness of collaborative care for the treatment of major depressive disorder in primary care. A systematic review. BMC Health Serv Res 2010;10:19.

13. Gilbody S, Bower P, Whitty P. Costs and consequences of enhanced primary care for depression: systematic review of randomised economic evaluations. Br J Psychiatry 2006;189:297-308.

14. Drummond M, Sculpher MJ, Torrance GW, et al. Methods for the economic evaluation of health care programmes. 4th edn. New York, United States: Oxford university press, 2015

15. Montgomery SA, Asberg M. A new depression scale designed to be sensitive to change. Br J Psychiatry 1979;134:382-9.

16. Weinstein MC, Torrance G, McGuire A. QALYs: the basics. Value Health 2009;12(Suppl 1):S5-S9.

17. Ramsey S, Willke R, Briggs A, et al. Good research practices for cost-effectiveness analysis alongside clinical trials: the ISPOR RCTCEA Task Force report. Value Health 2005;8:521-33.

18. Arnau RC, Meagher MW, Norris MP, et al. Psychometric evaluation of the beck depression inventory-II with primary care medical patients. Health Psychol 2001;20:112-9.

19. The EuroQol Group. EuroQol - a new facility for the measurement of health-related quality of life. Health Policy 1990;16:199-208.
20. The Swedish Pharmaceutical Industry Association's Service (LIF). FASSAllmänhet. Fass-verksamheten: Stockholm, 2017. http://www. fass.se/LIF/startpage.

21. Stewart WF, Ricci JA, Chee E, et al. Cost of lost productive work time among US workers with depression. JAMA 2003;289:3135-44.

22. Grochtdreis T, Brettschneider $\mathrm{C}$, Wegener $\mathrm{A}$, et al. Costeffectiveness of collaborative care for the treatment of depressive disorders in primary care: a systematic review. PLoS One 2015;10:e0123078.

23. Richards DA, Bower P, Chew-Graham C, et al. Clinical effectiveness and cost-effectiveness of collaborative care for depression in UK primary care (CADET): a cluster randomised controlled trial. Health Technol Assess 2016;20:1-192.

24. Aragonès E, López-Cortacans G, Sánchez-Iriso E, et al. Costeffectiveness analysis of a collaborative care programme for depression in primary care. J Affect Disord 2014;159:85-93.

25. Gensichen J, von Korff M, Peitz M, et al. Case management for depression by health care assistants in small primary care practices: a cluster randomized trial. Ann Intern Med 2009;151:369-78.

26. Swedish Council on Health Technology Assessment. SBU Book of methods Economic Evaluations. http://www.sbu.se/globalassets/ ebm/metodbok/sbushandbok_kapitel11.pdf. (accessed 30 Jan 2018). 